\title{
Comparison of ocular biometric measurements in patients with cataract using three swept-source optical coherence tomography devices
}

Richul Oh' ${ }^{1}$, Joo Youn Oh ${ }^{1,2,3}$, Hyuk Jin Choi ${ }^{1,2,3,4}$, Mee Kum Kim ${ }^{1,2,3}$ and Chang Ho Yoon ${ }^{1,2,3^{*}}$

\begin{abstract}
Background: Precise measurement of ocular biometry is critical for determining intraocular lens power. Newly developed swept-source optical coherence tomography (SS-OCT) - based ocular biometric devices, ANTERION and CASIA2 provide ocular biometric measurements as IOLMaster 700. This study aimed to assess agreement between three devices.

Methods: This retrospective comparative study includes patients with cataract who underwent ocular biometric measurements with three devices, ANTERION, CASIA2, and IOLMaster 700, at Seoul National University Hospital, in April 2020. Anterior keratometry, total keratometry, central corneal thickness (CCT), anterior chamber depth (ACD), lens thickness (LT), and axial length (AL) were the main parameters for the comparison. To assess the agreement between the devices, intraclass coefficient (ICC) and Bland-Altman analysis with 95\% limits of agreement (LoA) were used.

Results: A total of 47 eyes of 29 patients were measured with three devices. Average anterior keratometry showed excellent agreement (ICC $\geq 0.989$ ), and the mean difference was less than $0.1 \mathrm{D}$. However, the ICC of the total average keratometry ranged from 0.808 to 0.952 , and the difference was more than $0.43 \mathrm{D}$. The AL measured by ANTERION and IOLMaster 700 showed excellent agreement $(I C C=0.999)$, and the mean difference was $0.005 \mathrm{~mm}$. The ANTERION and IOLMaster 700 did not obtain AL in six (12.8\%) and three (6.4\%) cases, respectively ( $P=0.001$ by Fisher's exact test). The CCT, ACD, and LT also showed excellent agreement (ICC > 0.9).

Conclusions: The new SS-OCT-based devices, ANTERION, and CASIA2 showed a good agreement with IOLMaster 700 in measuring ocular biometry except for the total keratometry. The AL of ANTERION and IOLMaster 700 showed excellent agreement.
\end{abstract}

Keywords: Ocular biometry, Cataract, Total keratometry, Swept-source optical coherence tomography devices, ANTERION, CASIA2, IOLMaster 700

\footnotetext{
* Correspondence: ifree7@gmail.com

'Department of Ophthalmology, Seoul National University Hospital, 101 Daehak-ro, Jongno-gu, Seoul 03080, South Korea

${ }^{2}$ Department of Ophthalmology, Seoul National University College of Medicine, Seoul, South Korea

Full list of author information is available at the end of the article
}

(c) The Author(s). 2021 Open Access This article is licensed under a Creative Commons Attribution 4.0 International License, which permits use, sharing, adaptation, distribution and reproduction in any medium or format, as long as you give appropriate credit to the original author(s) and the source, provide a link to the Creative Commons licence, and indicate if changes were made. The images or other third party material in this article are included in the article's Creative Commons licence, unless indicated otherwise in a credit line to the material. If material is not included in the article's Creative Commons licence and your intended use is not permitted by statutory regulation or exceeds the permitted use, you will need to obtain permission directly from the copyright holder. To view a copy of this licence, visit http://creativecommons.org/licenses/by/4.0/. The Creative Commons Public Domain Dedication waiver (http://creativecommons.org/publicdomain/zero/1.0/) applies to the data made available in this article, unless otherwise stated in a credit line to the data. 


\section{Background}

In modern cataract surgery, it is important not only to remove the cataract but also to achieve accurate postoperative refractive error. The higher the generation of the intraocular lens (IOL) calculation formula, the higher the accuracy, and more ocular biometric parameters are required [1]. In this regard, precise measurement of ocular biometry is critical for determining the power of IOLs [2]. Recently, ocular biometry measurement devices with principles of swept-source optical coherence tomography (SS-OCT) were developed. SS-OCT devices use $1000-1350 \mathrm{~nm}$ of wavelength. They can provide a whole image from the cornea to the posterior lens. They are known to have a superior ability to successfully measure the axial length (AL) compared with a partial coherence interferometry device [3, 4]. IOLMaster 700 (Carl Zeiss Meditec), the first SS-OCT-based biometric device, is one of the most widely used devices for cataract surgery [3]. Many studies have shown that IOLMaster 700 had good agreement with other devices, including IOLMaster 500 [5-7].

ANTERION (Heidelberg Engineering) and CASIA2 (Tomey) are newly developed SS-OCT devices. ANTE RION uses a $1300 \mathrm{~nm}$ central wavelength of light. It provides a scan depth range of $32 \mathrm{~mm}$ for the axial length and an in-tissue axial resolution of $<10 \mu \mathrm{m}$. It uses OCT-based structural images to generate ocular biometric measurements [8]. CASIA2, an advanced version of CASIA SS-1000, uses a $1310 \mathrm{~nm}$ wavelength light with a scan speed of 50,000 A scans per second. CASIA2 has a scan range of $13 \mathrm{~mm}$ depth and $16 \mathrm{~mm}$ width [9]. They both provide ocular biometric measurements, including the anterior chamber depth (ACD), lens thickness (LT), and corneal keratometry. In addition, ANTERION with its deeper scan range, provides measurement of AL. They generally showed good repeatability and agreement with the IOLMaster 700 device [8-11]. However, so far, no previous studies have compared ANTERION and CASIA2 or all three devices in patients with cataract. This study aimed to assess the agreement between three devices, ANTERION, CASIA2, and IOLMaster 700, in terms of ocular biometry.

\section{Methods}

This retrospective study comprised patients with cataracts from Seoul National University Hospital (SNUH), Seoul, Republic of Korea, in April 2020. All procedures were conducted following the tenets of the Declaration of Helsinki, and the study design was approved by the Institutional Review Board of SNUH (IRB No. 2006-0261130). Owing to the retrospective design of the study and the use of deidentified patient information, the review board waived the need for written informed consent.
All patients underwent a standard test for cataract surgery according to the SNUH preoperative cataract examination protocol. The standard test for cataract surgery includes measurements using the three SS-OCT devices, specular microscopy, macular OCT, corneal topography, ultrasound A-scan, and automated keratometry. Among them, optical biometric measurements using the three SS-OCT devices were conducted before the other examinations. Patients who were diagnosed with retinal diseases, such as epiretinal membrane, age-related macular degeneration, or corneal diseases such as corneal opacity, keratoconus, and pterygium were excluded from this study. Patients who had already undergone refractive surgery were also excluded. Ocular biometry measurements, including both anterior and total keratometry, central corneal thickness (CCT), ACD, and LT, were obtained by ANTERION, CASIA2, and IOLMaster 700. AL was measured only by ANTERION and IOLMaster 700 because CASIA2 does not provide AL measurement. ACD was defined as the axial distance from the corneal endothelium to the lens. Because IOLMaster 700 measures the ACD from the corneal epithelium to the anterior lens surface, we subtracted the CCT value from the ACD measured by the IOLMaster 700 .

Ocular biometric evaluation proceeded in the order of ANTERION, CASIA2, and IOLMaster 700. Measurements were performed by one examination specialist. The three devices were lined up in the same examination room. We confirmed that every time we examined the patient, the room was constantly illuminated under $10 \mathrm{~lx}$, as measured with a light meter (LX-1102, Lutron, Taiwan).

All statistical analyses were performed using $\mathrm{R}$ software ( $\mathrm{R}$ version 4.0.2. Available at http://www.r-project. org; accessed June 2020). Continuous variables are presented as mean \pm standard deviation. Acquisition rates of AL measurements were analyzed by Fisher's exact test. To assess the agreement between the measurements of the devices, intraclass correlation coefficients (ICC, twoway random, single measure) were calculated [12]. ICC was regarded as follows: $<0.75$, poor to moderate reliability; 0.75-0.90, good reliability; and $>0.90$, excellent reliability [11]. Bland-Altman analysis with $95 \%$ limits of agreement (LoA) was also used for all pairs. The level of statistical significance was set at $P<.05$. Due to the retrospective design of this study, the sample size was not calculated.

\section{Results}

A total of 47 eyes of 29 patients with cataract (14 males, 15 females) were included in this study, and their mean age was $64.41 \pm 10.68$ (range, $31-83$ years). Table 1 shows the descriptive summary of the ocular biometric measurements taken by the three devices and their ICC 
Table 1 Summary of the ocular biometric measurements measured by ANTERION, CASIA2, and IOLMaster 700

\begin{tabular}{|c|c|c|c|c|c|c|c|c|c|}
\hline & \multirow[t]{2}{*}{ ANTERION } & \multirow[t]{2}{*}{ CASIA2 } & \multirow[t]{2}{*}{ IOLMaster 700} & \multicolumn{2}{|c|}{ ANTERION vs IOLMaster 700} & \multicolumn{2}{|c|}{ CASIA2 vs IOLMaster 700} & \multicolumn{2}{|c|}{ ANTERION vs CASIA2 } \\
\hline & & & & ICC & Difference & ICC & Difference & ICC & Difference \\
\hline Anterior average $\mathrm{K}, \mathrm{D}$ & $43.92 \pm 1.62$ & $44.02 \pm 1.57$ & $43.98 \pm 1.63$ & 0.991 & $-0.059 \pm 0.213$ & 0.989 & $0.038 \pm 0.233$ & 0.990 & $-0.097 \pm 0.201$ \\
\hline Anterior steep K, D & $44.42 \pm 1.62$ & $44.51 \pm 1.55$ & $44.38 \pm 1.60$ & 0.974 & $0.034 \pm 0.367$ & 0.959 & $0.13 \pm 0.439$ & 0.962 & $-0.096 \pm 0.433$ \\
\hline Anterior flat $K, D$ & $43.42 \pm 1.70$ & $43.53 \pm 1.66$ & $43.58 \pm 1.68$ & 0.976 & $-0.166 \pm 0.334$ & 0.978 & $-0.059 \pm 0.348$ & 0.970 & $-0.107 \pm 0.403$ \\
\hline Anterior cylinder K, D & $1.01 \pm 0.71$ & $0.99 \pm 0.76$ & $0.80 \pm 0.46$ & 0.598 & $0.21 \pm 0.516$ & 0.443 & $0.189 \pm 0.653$ & 0.526 & $0.021 \pm 0.719$ \\
\hline Total average K, D & $43.33 \pm 1.63$ & $42.89 \pm 1.52$ & $43.94 \pm 1.61$ & 0.922 & $-0.61 \pm 0.255$ & 0.808 & $-1.049 \pm 0.237$ & 0.952 & $0.439 \pm 0.232$ \\
\hline Total steep K, D & $43.85 \pm 1.64$ & $43.36 \pm 1.57$ & $44.37 \pm 1.64$ & 0.920 & $-0.525 \pm 0.425$ & 0.801 & $-1.016 \pm 0.443$ & 0.918 & $0.491 \pm 0.453$ \\
\hline Total flat K, D & $42.81 \pm 1.69$ & $42.42 \pm 1.56$ & $43.51 \pm 1.62$ & 0.895 & $-0.708 \pm 0.359$ & 0.790 & $-1.094 \pm 0.353$ & 0.938 & $0.386 \pm 0.438$ \\
\hline Total cylinder K, D & $1.04 \pm 0.74$ & $0.94 \pm 0.73$ & $0.86 \pm 0.47$ & 0.505 & $0.183 \pm 0.602$ & 0.441 & $0.078 \pm 0.649$ & 0.461 & $0.105 \pm 0.761$ \\
\hline $\mathrm{AL}^{\mathrm{a}}, \mathrm{mm}$ & $24.00 \pm 1.60$ & NA & $23.99 \pm 1.54$ & 0.999 & $-0.005 \pm 0.053$ & NA & NA & NA & NA \\
\hline $\mathrm{CCT}, \mu \mathrm{m}$ & $535.89 \pm 34.29$ & $533.60 \pm 34.26$ & $535.19 \pm 35.04$ & 0.994 & $0.702 \pm 3.735$ & 0.986 & $-1.596 \pm 5.57$ & 0.981 & $2.298 \pm 6.304$ \\
\hline $\mathrm{ACD}, \mathrm{mm}$ & $2.62 \pm 0.39$ & $2.60 \pm 0.48$ & $2.53 \pm 0.47$ & 0.990 & $0.058 \pm 0.033$ & 0.982 & $0.075 \pm 0.052$ & 0.994 & $-0.015 \pm 0.049$ \\
\hline $\mathrm{LT}^{\mathrm{b}}, \mathrm{mm}$ & $4.63 \pm 0.43$ & $4.60 \pm 0.55$ & $4.54 \pm 0.52$ & 0.915 & $0.154 \pm 0.159$ & 0.963 & $0.088 \pm 0.123$ & 0.961 & $0.064 \pm 0.141$ \\
\hline
\end{tabular}

Continuous data are presented as mean \pm standard deviation

Differences between two devices were defined by subtracting the latter value from the former value

$A C D$ anterior chamber depth, $A L$ axial length, $C C T$ central corneal thickness, $D$ Diopter, ICC intraclass correlation coefficient, $K$ keratometry, $L T$ lens thickness, NA not available, vs versus

${ }^{a} \mathrm{AL}$ was analyzed with 41 eyes that could be measured by both ANTERION and IOLMaster 700

bT was analyzed with 44 eyes that could be measured by all three devices

values. For most of the parameters, ICC values between the devices were greater than 0.90 . Bland-Altman plots for agreement analysis between the devices are presented in Figs. 1, 2 and 3 (Fig. 1, ANTERION vs. Master 700; Fig. 2, CASIA2 vs. Master 700; Fig. 3, ANTERION vs. CASIA2).

In the measurement of anterior keratometry, ANTE RION and CASIA2 showed excellent agreement with IOLMaster 700 (ICC value of 0.991 , and 0.989 , respectively). Anterior keratometry measured by ANTERION and CASIA2 had an ICC value of 0.990 . The mean differences were less than $0.060 \mathrm{D}$.

In the measurement of total keratometry, ANTERION and CASIA2 had the greatest ICC values (0.952). While ANTERION and IOLMaster 700 had an ICC value of 0.922 , CASIA2 and IOLMaster 700 had an ICC value of 0.808 . The mean difference in total keratometry between CASIA2 and IOLMaster 700, ANTERION and IOLMaster 700, and ANTERION and CASIA2 was 1.049 D, 0.61 $\mathrm{D}$, and $0.439 \mathrm{D}$, respectively.

$\mathrm{AL}$ measurements were not obtained in three eyes with the IOLMaster 700 and six eyes with the ANTE RION ( $P=0.001$ by Fisher's exact test). Both IOLMaster 700 and ANTERION failed to measure AL in three eyes. Lens grading of these three eyes were intumescent, NO6, and P5 according to the LOCS III classification [13]. In addition, ANTERION also failed to measure AL in other three eyes, of which ALs were successfully obtained by IOLMaster 700 . The lens grade of these eyes was $\mathrm{C} 1 \mathrm{NO} 1, \mathrm{P} 4$, and anterior subcapsular opacity (ASC).

In the measurement of ACD, new SS-OCT devices, ANTERION, and CASIA2 had the greatest ICC value (0.994). Each of them had a comparably great agreement with the IOLMaster 700 (ICC value of $0.990,0.982$, relatively).

CCT and ACD also showed excellent agreement among the three devices. The mean differences of CCT and ACD between the devices were less than $3 \mu \mathrm{m}$ and $0.08 \mathrm{~mm}$, respectively. The Bland-Altman plot of CCT and $\mathrm{ACD}$ showed that most differences were within $10 \mu \mathrm{m}$ and $0.15 \mathrm{~mm}$, respectively.

In the measurement of LT, CASIA2 measured LT in all eyes. However, ANTERION and IOLMaster 700 failed to measure LT in four and two eyes, respectively. For one eye with ASC, both ANTERION and IOLMaster 700 failed to measure LT. With the eyes with measurable LT in all devices, they also showed excellent agreement with each other, as shown in Table 1.

\section{Discussion}

In this study, we evaluated the agreement of ocular biometric measurements in patients with cataracts among the three SS-OCT devices. To the best of our knowledge, this is the first study to compare the three devices simultaneously. In addition, for the first time, we compared the total corneal power values that account for posterior corneal curvature.

Generally, anterior keratometry measurements showed excellent agreement. We found the difference of anterior average $\mathrm{K}$ of each device was less than $0.1 \mathrm{D}$ (ANTE RION vs. IOLMaster 700, -0.059 D; CASIA2 vs. IOLMaster 700, $0.038 \mathrm{D}$; and ANTERION vs. CASIA2, $0.097 \mathrm{D})$, which seems to be clinically insignificant. This is in line with the study by Fisus et al. [8] that the mean 

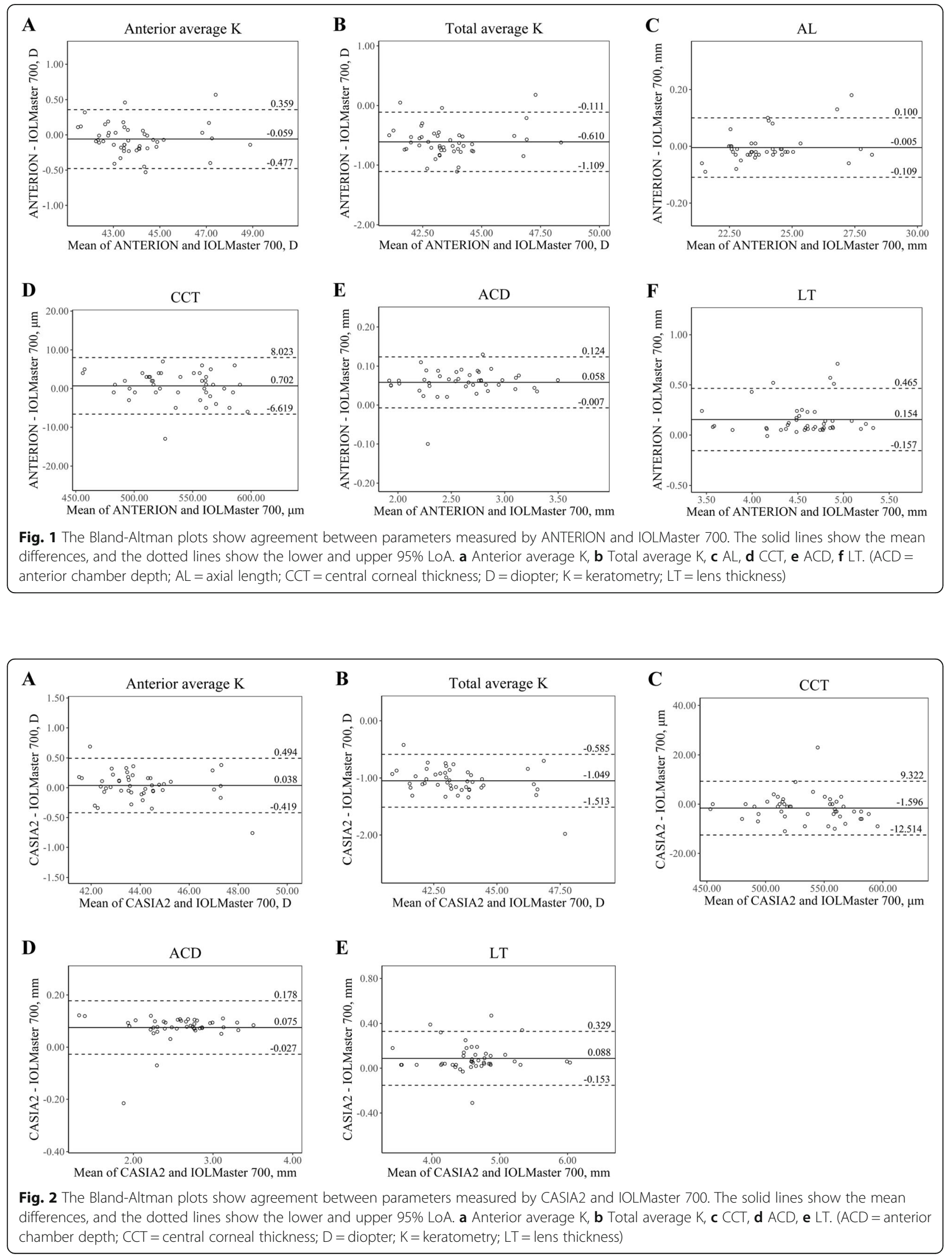

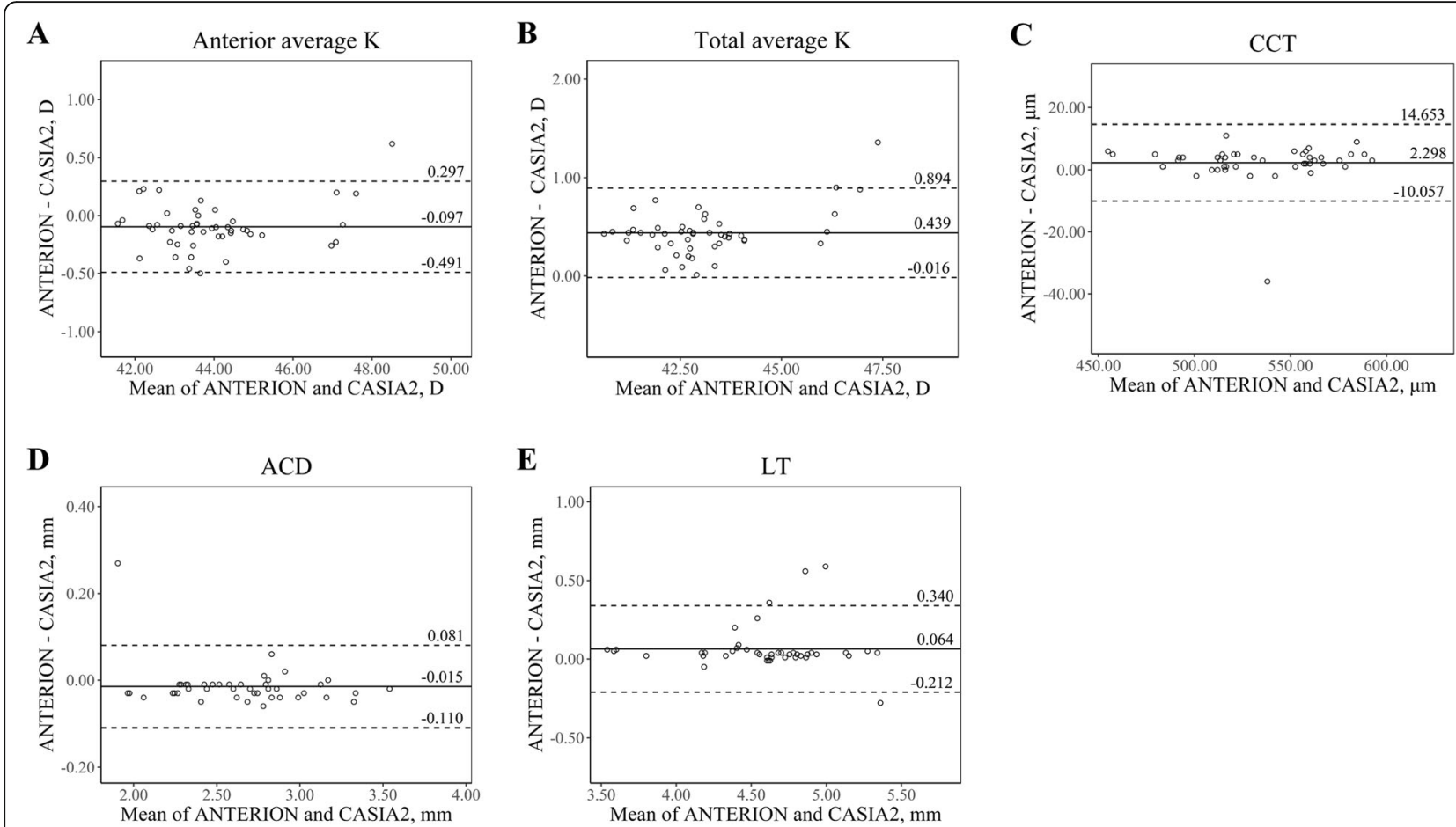

Fig. 3 The Bland-Altman plots show agreement between parameters measured by ANTERION and CASIA2. The solid lines show the mean differences, and the dotted lines show the lower and upper 95\% LoA. a Anterior average $K$, b Total average $K, \mathbf{c} C C T$, $\mathbf{d} A C D$, e LT. (ACD = anterior chamber depth; $C C T=$ central corneal thickness; $\mathrm{D}=$ diopter; $\mathrm{K}=$ keratometry; $\mathrm{LT}=$ lens thickness)

absolute difference was 0.04 D when comparing ANTE RION and IOLMaster 700.

Regarding total keratometry, a better refractive outcome was achieved using total keratometry compared to conventional anterior keratometry in the calculation of IOLs for cataract surgery by IOLMaster 700 [14-16]. In addition, it is expected that more patients will undergo cataract surgery who have previously undergone refractive surgery; therefore, it will become more important to accurately measure total corneal power [15, 17]. However, the total average keratometry measured by the three devices showed a difference of $0.439 \mathrm{D}$ or more. ICC values for total average keratometry were lower than those of anterior average keratometry, and the Bland-Altman plot of total average keratometry showed a wider 95\% LoA than that of average anterior keratometry. The corneal power difference of $0.439 \mathrm{D}$ is about $0.64 \mathrm{D}$ in the IOL plane, assuming the IOL plane to the corneal plane equivalent to the power conversion factor is about 0.69 [18]. Considering that the $0.5 \mathrm{D}$ is the currently used IOL power step, the total keratometry values derived from each device should not be interchanged when calculating IOLs. The discrepancy of the three devices in total keratometry could be caused by the different algorism for calculating total keratometry in each device $[5,8,19]$. IOLMaster 700 obtains anterior keratometry with 18 reflected spots in hexagonal patterns at three zones $(1.5 \mathrm{~mm}, 2.5 \mathrm{~mm}$, and $3.5 \mathrm{~mm})$ and posterior keratometry with SS-OCT tomography [16, 20]. Meanwhile, ANTERION and CASIA2 calculate anterior and total keratometry only with SS-OCT images (ANTE RION: 65 B-scans with 256 A-scans each; CASIA2: 16 B-scans with 800 A-scans each) [8, 21].

We compared AL measured by only ANTERION with IOLMaster 700, because CASIA2 does not provide AL. It is known that the interdevice agreement of AL measured by optical method is excellent [22]. We also found ANTERION with IOLMaster 700 had an excellent agreement in measuring AL, with an ICC value of 0.999 , and the average difference was $0.005 \mathrm{~mm}$. Accounting that a measurement error of $1 \mathrm{~mm}$ of AL induces $2.5 \mathrm{D}$ deviation in IOL calculation in the eye with an average AL $(23.5 \mathrm{~mm})$, those $0.005 \mathrm{~mm}$ of AL difference result in $0.0125 \mathrm{D}$ of IOL power difference, and it seems that the AL by ANTERION and IOL Master 700 could be interchangeable [23]. AL measurement by optical biometry has been shown to fail in eyes with dense or posterior capsular cataract [5]. In this study, AL of six and three eyes were not obtained by ANTERION and IOLMaster 700 , respectively, and most of their cataract status was dense nucleus cataract or posterior or anterior subcapsular cataract. ANTERION showed significantly higher $\mathrm{AL}$ measurement failure than IOLMaster 700 . This result was unexpected because longer wavelengths 
improve penetration, and ANTERION (1300 nm) uses a longer wavelength than IOLMaster $700(1050 \mathrm{~nm})$ for measuring AL [24]. The different acquisition methods could be the cause of this discrepancy. IOLMaster 700 measures AL by the average values of three scans in each of the six meridians [5]. ANTERION obtains AL by averaging three consecutive three subsets of data.

In the measurement of CCT, ACD, and LT, the three devices showed excellent agreement. The 95\% LoAs were narrow and clinically insignificant. Regarding CCT, it is also important for screening before refractive surgery. In our study, which included only normal corneas, CCT seems to be interchangeable; however, further study is needed for pathologic conditions such as keratoconus or post-refractive surgery patients.

This study has several limitations. First, the sample size was relatively small. Second, all included patients who had cataracts. Since cataracts can affect optical physics during measurements, there might be some biases. Further studies with normal eyes will provide more information about the agreements between these devices. Third, all patients were Asian. Therefore, our data may not be generalizable to other ethnicities. Finally, we did not use a randomization sequence when measuring the three machines. However, they all are non-contact devices, and it can be assumed that the order of measurements would not affect ocular biometric values.

\section{Conclusions}

SS-OCT devices, ANTERION, CASIA2, and IOL Master 700 showed good agreement in parameters of anterior corneal curvature, CCT, ACD, and LT. AL of ANTE RION and IOLMaster 700 showed excellent agreement, and it seems to be interchangeable. However, the total keratometry value of each device was different and should not be used interchangeably.

\section{Abbreviations}

ACD: Anterior chamber depth; ASC: Anterior subcapsular opacity; AL: Axial length; CCT: Central corneal thickness; ICC: Intraclass correlation coefficients; IOL: Intraocular lens; LT: Lens thickness; LoA: Limits of agreement; SSOCT: Swept-source optical coherence tomography

\section{Acknowledgments}

Not applicable.

\section{Authors' contributions}

$\mathrm{RO}$ acquired and analyzed the data, drafted the initial manuscript, and revised the manuscript. JYO, HJC, MKK conceptualized and designed the study, and collected data. CHY conceptualized and designed the study, coordinated and supervised data collection, analyzed the data, critically reviewed the manuscript, and revised the manuscript. All authors read and approved the final manuscript

\section{Funding}

None.

\section{Availability of data and materials}

The datasets used and analyzed during the current study are available from the corresponding author on reasonable request.

\section{Ethics approval and consent to participate}

This study was approved by the Seoul National University College of Medicine/Seoul National University Hospital Institutional Review Board (No. 2006-026-1130) and was conducted in accordance with the Declaration of Helsinki. The need for written informed consent was waived because of the retrospective design and the use of deidentified patient data.

\section{Consent for publication}

Not applicable.

\section{Competing interests}

The authors declare they have no competing interest.

\section{Author details}

'Department of Ophthalmology, Seoul National University Hospital, 101 Daehak-ro, Jongno-gu, Seoul 03080, South Korea. ${ }^{2}$ Department of Ophthalmology, Seoul National University College of Medicine, Seoul, South Korea. ${ }^{3}$ Laboratory of Ocular Regenerative Medicine and Immunology (LORMI), Artificial Eye Center, Seoul National University Hospital Biomedical Research Institute, Seoul, South Korea. ${ }^{4}$ Department of Ophthalmology, Seoul National University Hospital Healthcare System Gangnam Center, Seoul, South Korea.

Received: 12 November 2020 Accepted: 14 January 2021

Published online: 27 January 2021

\section{References}

1. Amro M, Chanbour W, Arej N, Jarade E. Third- and fourth-generation formulas for intraocular lens power calculation before and after phakic intraocular lens insertion in high myopia. J Cataract Refract Surg. 2018;44: $1321-5$.

2. Olsen T. Calculation of intraocular lens power: a review. Acta Ophthalmol Scand. 2007:85:472-85.

3. Yang CM, Lim DH, Kim HJ, Chung TY. Comparison of two swept-source optical coherence tomography biometers and a partial coherence interferometer. PLoS One. 2019;14:e0223114.

4. Fukuda S, Ueno Y, Fujita A, Mori H, Tasaki K, Murakami T, et al. Comparison of anterior segment and lens biometric measurements in patients with cataract. Graefes Arch Clin Exp Ophthalmol. 2020;258:137-46.

5. Akman A, Asena L, Gungor SG. Evaluation and comparison of the new swept source OCT-based IOLMaster 700 with the IOLMaster 500. Br J Ophthalmol. 2016;100:1201-5.

6. Arriola-Villalobos P, Almendral-Gomez J, Garzon N, Ruiz-Medrano J, Fernandez-Perez C, Martinez-de-la-Casa JM, et al. Agreement and clinical comparison between a new swept-source optical coherence tomographybased optical biometer and an optical low-coherence reflectometry biometer. Eye (Lond). 2017;31:437-42.

7. Kunert KS, Peter M, Blum M, Haigis W, Sekundo W, Schutze J, et al. Repeatability and agreement in optical biometry of a new swept-source optical coherence tomography-based biometer versus partial coherence interferometry and optical low-coherence reflectometry. J Cataract Refract Surg. 2016;42:76-83.

8. Fisus AD, Hirnschall ND, Findl O. Comparison of 2 swept-source optical coherence tomography-based biometry devices. J Cataract Refract Surg. 2021;47:87-92.

9. Sato T, Yamamoto J, Shibata S, Hayashi K. Comparison of anterior segment measurements obtained by different swept-source OCT-based biometers. Can J Ophthalmol. 2020;55:e165-e8.

10. Tañá-Rivero P, Aguilar-Córcoles S, Ruiz-Mesa R, Montés-Micó R. Repeatability of whole-cornea measurements using a new swept-source optical coherence tomographer. Eur J Ophthalmol. 2020;1120672120944022.

11. Shoji T, Kato N, Ishikawa S, Ibuki H, Yamada N, Kimura I, et al. In vivo crystalline lens measurements with novel swept-source optical coherent tomography: an investigation on variability of measurement. BMJ Open Ophthalmol. 2017;1:e000058.

12. Morrow JR Jr, Jackson AW. How "significant" is your reliability? Res Q Exerc Sport. 1993;64:352-5. 
13. Chylack LT Jr, Wolfe JK, Singer DM, Leske MC, Bullimore MA, Bailey IL, et al. The lens opacities classification system III. Arch Ophthalmol. 1993;111:831-6.

14. Srivannaboon S, Chirapapaisan C. Comparison of refractive outcomes using conventional keratometry or total keratometry for IOL power calculation in cataract surgery. Graefes Arch Clin Exp Ophthalmol. 2019;257:2677-82.

15. Lawless M, Jiang JY, Hodge C, Sutton G, Roberts TV, Barrett G. Total keratometry in intraocular lens power calculations in eyes with previous laser refractive surgery. Clin Exp Ophthalmol. 2020:48:749-56.

16. Fabian $E$, Wehner W. Prediction accuracy of total keratometry compared to standard keratometry using different intraocular lens power formulas. J Refract Surg. 2019;35:362-8.

17. Hamilton DR, Hardten DR. Cataract surgery in patients with prior refractive surgery. Curr Opin Ophthalmol. 2003;14:44-53.

18. Goggin M, Moore S, Esterman A. Toric intraocular lens outcome using the manufacturer's prediction of corneal plane equivalent intraocular lens cylinder power. Arch Ophthalmol. 2011;129:1004-8.

19. Wylegala A, Mazur R, Bolek B, Wylegala E. Reproducibility, and repeatability of corneal topography measured by Revo NX, Galilei G6 and Casia 2 in normal eyes. PLoS One. 2020;15:e0230589.

20. Liao X, Peng Y, Liu B, Tan QQ, Lan CJ. Agreement of ocular biometric measurements in young healthy eyes between IOLMaster 700 and OA-2000 Sci Rep. 2020;10:3134.

21. Zhang T, Zhou Y, Young CA, Chen A, Jin G, Zheng D. Comparison of a new swept-source anterior segment optical coherence tomography and a scheimpflug camera for measurement of corneal curvature. Cornea. 2020;39: 818-22.

22. Huang J, Chen H, Li Y, Chen Z, Gao R, Yu J, et al. Comprehensive comparison of axial length measurement with three swept-source OCTbased biometers and partial coherence interferometry. J Refract Surg. 2019; 35:115-20.

23. Wilson ME, Trivedi RH. Axial length measurement techniques in pediatric eyes with cataract. Saudi J Ophthalmol. 2012;26:13-7.

24. Hirnschall N, Varsits R, Doeller B, Findl O. Enhanced penetration for axia length measurement of eyes with dense cataracts using swept source optical coherence tomography: a consecutive observational study. Ophthalmol Ther. 2018;7:119-24.

\section{Publisher's Note}

Springer Nature remains neutral with regard to jurisdictional claims in published maps and institutional affiliations.

Ready to submit your research? Choose BMC and benefit from:

- fast, convenient online submission

- thorough peer review by experienced researchers in your field

- rapid publication on acceptance

- support for research data, including large and complex data types

- gold Open Access which fosters wider collaboration and increased citations

- maximum visibility for your research: over $100 \mathrm{M}$ website views per year

At $\mathrm{BMC}$, research is always in progress.

Learn more biomedcentral.com/submissions 